\title{
MODE SUPERPOSITION IN MULTI-DEGREE OF FREEDOM SYSTEMS USING EARTHQUAKE RESPONSE SPECTRUM DATA
}

\author{
By H. C. Merchant and D. E. Hudson
}

\begin{abstract}
Dynamic responses of a series of typical three-degree of freedom structures to strong-motion earthquake excitation were calculated by analog computer techniques and were compared with approximate responses obtained by a superposition of individual modes derived from response spectrum curves. The results indicate that a suitably weighted average of the sum of the absolute values of the individual modes and the square root of the sum of the squares of the modes will give a practical design criterion for the base shear forces in multi-story buildings. For critical designs, this weighted average reduces to the absolute sum of the modes, which is found to be close to the true value for a significantly high proportion of typical earthquake-structure combinations.
\end{abstract}

\section{INTRODUCTTON}

The calculation of the response of an actual structure to an earthquake exciting force is a problem which is simple in principle but is complicated in practice because of the large number of parameters involved and the lengthy numerical computations required. The introduction of the concept of the response spectrum by Benioff (1934) and Biot (1941) was an effort to reduce the number of basic parameters for such dynamic problems. The response spectrum provides, in effect, a way of separating that part of the response calculation which depends upon the particular earthquake exciting force from that part which involves mainly the properties of the structure.

The maximum relative velocity response spectrum is defined as the maximum relative velocity in a single degree of freedom, linear, spring-mass-dashpot system acted upon by a prescribed force-time relationship, plotted for various values of natural frequency or period and damping. Relative displacements and hence structural strains can be expressed in terms of the velocity spectrum through simple approximate relationships (Hudson, 1956). By limiting the response spectrum concept to the linear single degree of freedom system which can be described in terms of natural frequency and an equivalent viscous friction, the parameters are reduced to a manageable number.

The response spectrum has a direct value as a description of the earthquake ground motion in terms that are significantly related to structural response. The response spectrum, for example, gives in effect a harmonic analysis of the earthquake ground motions, and serves the same purpose in defining significant period content as would the related Fourier spectrum.

It is not to be expected that the response spectrum alone will give a complete description of the responses of complicated structures, since such structures cannot be even approximately represented as a single degree of freedom system. It does happen, however, that a number of types of structures, such as elevated water tanks and small building structures of simple form, do behave essentially as single degree of freedom systems for typical earthquake excitations. For such structures the response spectrum gives at once the information required by the design engineer. 
For other common types of structures, such as tall buildings, chimneys, towers, etc., it is well known that the contributions of the higher modes of vibration to the earthquake response may be far from negligible. It is thus of interest to investigate ways of extending response spectrum techniques to improve the accuracy of the design calculations for these more complicated structures for which the higher modes must be considered.

The basic difficulty involved in this extension is illustrated in fig. 1, which shows the responses of the individual modes of vibration of a structure to a particular

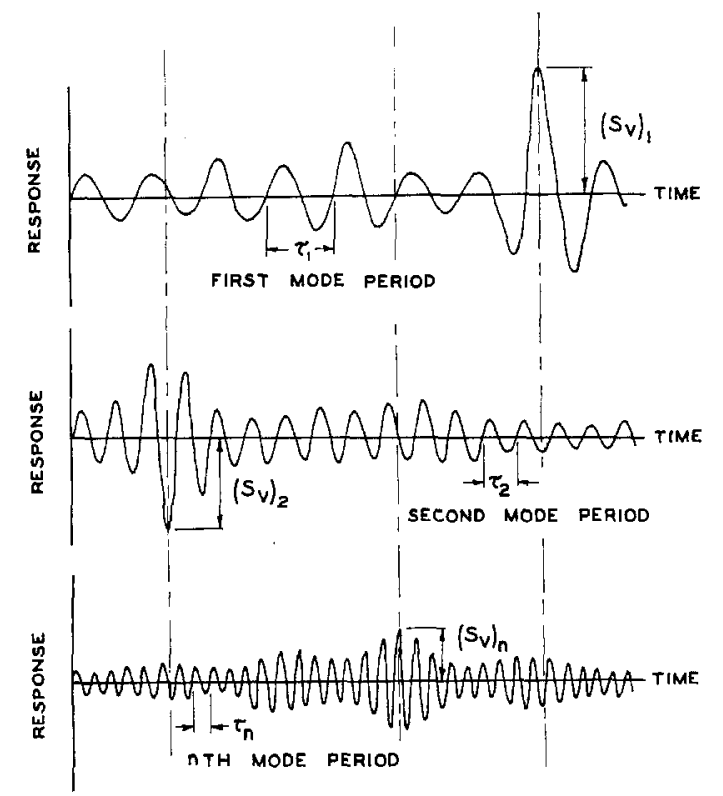

Fig. 1. Mode superposition in multi-degree of freedom systems.

earthquake ground motion. The complete response would be obtained by combining all of the modes with appropriate participation factors depending upon the mode shapes and mass distributions of the structure. The combined effects of all of the modes could thus be computed as a function of time, and it would be found that at one particular time a maximum combined response would be obtained.

The response spectrum curves give the relationship between the period $\tau$ and the maximum value of the response at that period, as indicated for the individual modes of figure 1 . The time at which this maximum occurs is not preserved in the spectrum curves. Since the maximum values of the individual modes will in general occur at different times for the different modes, the phase relationships between the maxima are lost, and hence the exact way in which the various modes combine cannot be determined.

The problem can now be stated in the following way: Given response spectrum curves for an earthquake, and knowing the natural periods and mode shapes for a structure, what is the most appropriate way of calculating an approximate value of maximum response, and what order of accuracy can be expected? What would be hoped for from the answer to this question would be a design technique which would 
ensure that the errors in the maximum response calculation would be kept below a prescribed level.

\section{Methods of Mode Combination}

The first suggestion that was made as a suitable method of mode combination was to take the sum of the absolute values of the individual modes. This idea was a part of Biot's original development (Biot, 1943). This absolute sum would evidently give the worst possible combination, and would thus set an upper bound on the response. Since it is unlikely that the maximum response values in the individual modes would all occur at the same time with the same sign, the true maximum response would usually be somewhat less than the absolute sum. A practical advantage of this method of combination is that any error is always on the safe side.

For pulse-type excitations it has been shown by Fung and Barton (1958) that the algebraic sum of the individual modes will give for relatively high-frequency responses a better value than the absolute sum. Their studies confirm, however, that for earthquake-like excitations, the absolute sum would be preferable.

Another suggestion that has been made is that a fixed percentage of the higher mode responses should be added to the first mode response, or that the first mode response be increased by a fixed percentage. These ideas have been examined for earthquake excitations by Clough (1955).

A different approach to the problem is based on ideas drawn from probability theory. Although the peak values of the individual mode responses occurring at different times cannot strictly speaking be treated in terms of simple statistics, it has been suggested by Rosenblueth (1956) that the mode responses might be combined as the square root of the sum of the squares to give a useful idea of the most probable combination. This criterion would evidently lead to lower values of total response than the absolute sum, and hence might be a more realistic assessment of average conditions.

A refinement of the above ideas that has also been suggested involves an average of the absolute sum and the square root of the sum of the squares (Jennings, 1958).

The object of the investigations reported in the present paper is to make a direct comparison of the various theories outlined above with exact solutions obtained for typical structures and typical earthquake excitations (Merchant, 1961).

\section{Calculation of Complete System Response}

Computations of the complete systems were made on an electronic differential analyzer which was designed for the purpose. The basic principle of this analog computer involves an adder-integrator circuit to simulate each mode of vibration of the multi-degree of freedom system, and a combination of the inputs and outputs of each of the modal circuits in such a way that the correct total response is produced. Figure 2 shows a schematic diagram of this special purpose analog computer.

Three modal circuits were provided for in the computer. This permits the exact solution of a three-degree of freedom system, or a three-mode approximate solution for more complicated systems. For the type of structures under consideration, past studies have shown that modes of vibration higher than the third do not usually contribute significantly to the total response. 
The earthquake input function was obtained from the same function generator that is regularly used with the electric analog response spectrum analyzer (Caughey et al, 1960). The accuracy of the earthquake function generation and of the total response computation is of the same order as that involved in the standard response spectrum calculations. Digital computer comparisons have shown that these accuracies of about $5 \%$ are as good as is consistent with the available strong-motion accelerograph data.

The performance and accuracy of the analog computer were checked by repeating several problems for which solutions were available from other investigations.

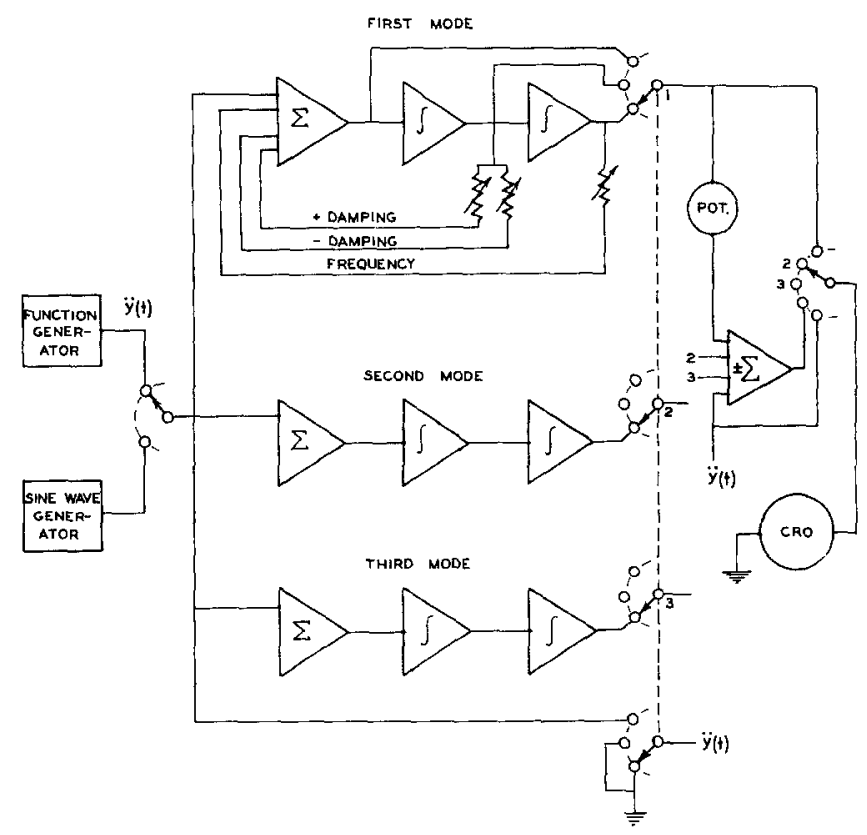

Frg. 2. Mode superposition with an electronic differential analyzer.

The published earthquake response spectrum curves for several earthquakes were checked, and the steady state response of a damped two-degree of freedom system to a sinusoidal exciting force was compared with an analytical solution. The response of the Alexander Building to the San Francisco earthquake of March 22, 1957, was checked against experimental measurements, (Hudson, 1960) and a three-mode approximation to an 8-story building was compared with digital computer results reported by Jennings (1958). All of these checks indicated that a satisfactory level of accuracy was being attained.

\section{Earthquakes and Structurad Types Investigated}

In order to study the mode superposition possibilities for a range of structural parameters and for various representative earthquakes, the following earthquakes and building types were studied.

Three typical earthquake ground acceleration records were selected. A relatively 
short time duration earthquake (approximately 5 seconds) was represented by the N 10 E component of the San Francisco Earthquake of March 22, 1957, recorded at Golden Gate Park (Hudson and Housner, 1959). As an earthquake of moderate magnitude and time duration, the $\mathrm{N} 21 \mathrm{E}$ component of the Taft Earthquake of July 21, 1952, was used (Murphy and Cloud, 1954; Housner, 1959). The third earthquake selected was the E-W component of the El Centro Earthquake of May 18, 1940, which has the largest ground acceleration magnitude yet recorded (Neumann, 1942; Housner et al, 1953). The above references to the various earth quakes include both the acceleration-time records and the response spectrum curves.

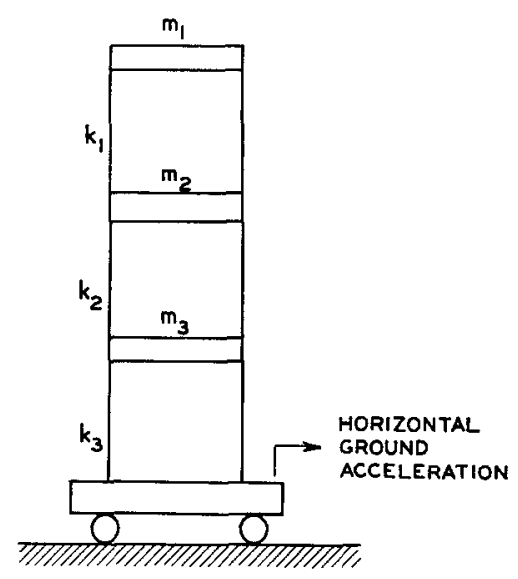

FIG. 3. Model of a shear-type structure subjected to earthquake ground acceleration.

Three building types were selected to cover a range of structural parameters typical of multi-story buildings. These three-degree of freedom, shear type buildings (fig. 3) may best be described by giving the mass and stiffness distributions and the ratios of the natural frequencies $\omega_{n}$

1. "Uniform Building"; $m_{1}=m_{2}=m_{3} ; k_{1}=k_{2}=k_{3}$

$$
\omega_{1}=\omega_{2} / 2.8=\omega_{3} / 4.05
$$

2. "Taper Building"; $\quad m_{2}=2 m_{1}, m_{3}=3 m_{1} ; k_{2}=2 k_{1}, k_{3}=3 k_{1}$

3. "Step Building"; $\quad m_{2}=m_{3}=2 m_{1} ; k_{2}=k_{3}=2 k_{1}$

$$
\omega_{1}=\omega_{2} / 2.30=\omega_{3} / 3.36
$$

One of the advantages of the particular type of computation used is that the damping in each mode can be independently set to any required amount. It is not necessary to consider the actual mechanism of the damping, or to decide whether the damping is primarily internal, involving relative motions between the floors, or external. Three distributions of damping were selected for the computations:

1. All modes, zero damping.

2. First mode, $2.5 \%$ critical; second and third mode, $5 \%$ critical.

3. All modes, $5 \%$ critical. 


\section{Results of the Calculations}

Abstracts of the computational results are presented in figures 4-8. In fig. 4 the uppermost diagram is the key to the results wherein it is shown that data are presented for five sets of structures having fundamental periods of $0.5,1.0,1.5,2.0$ and 2.5 seconds respectively. For each of these periods there is plotted the maximum
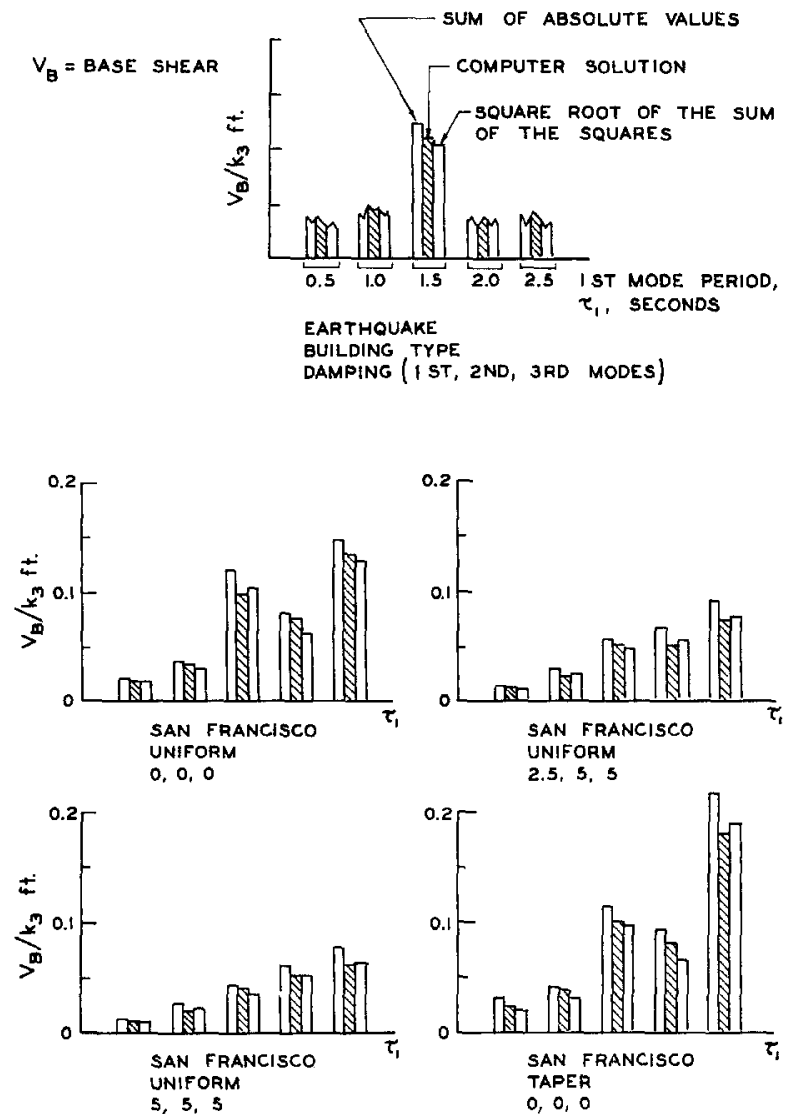

FIG. 4, Base shear comparisons San Francisco earthquake.

value of the base shear divided by the first-story spring constant $\left(V_{B} \div k_{3}\right)$, which is thus a plot of the relative displacement in the first story. For each period the maximum value of $\left(V_{B} \div k_{3}\right)$ is plotted for three cases, (1) the sum of the absolute values of the modes, (2) the "exact" computer solution shown cross-hatched, and (3) the square root of the sum of the squares of the modal values. Beneath each diagram there is listed the earthquake ground acceleration used to excite the structures, the type of building, and the percent of critical damping in each mode. Data is presented for nine combinations of earthquake, building type, and damping. It is seen in figs. 4-8 that the sum of the absolute values is always larger than the "exact" computer solution which is as it should be. The square root of the sum of the squares is sometimes larger and sometimes smaller than the "exact" solution. The percentage variations between the three methods of computation do not appear to be 

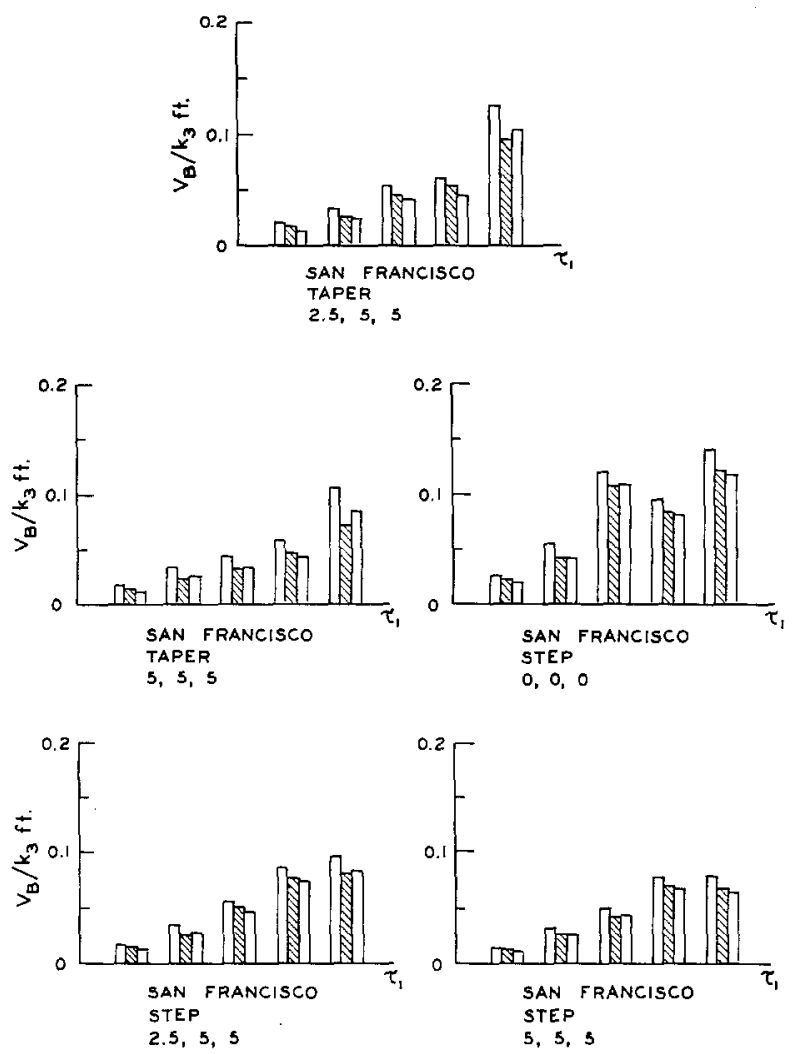

Fig. 5. Base shear comparisons (see fig. 4) San Francisco earthquake (cont.).
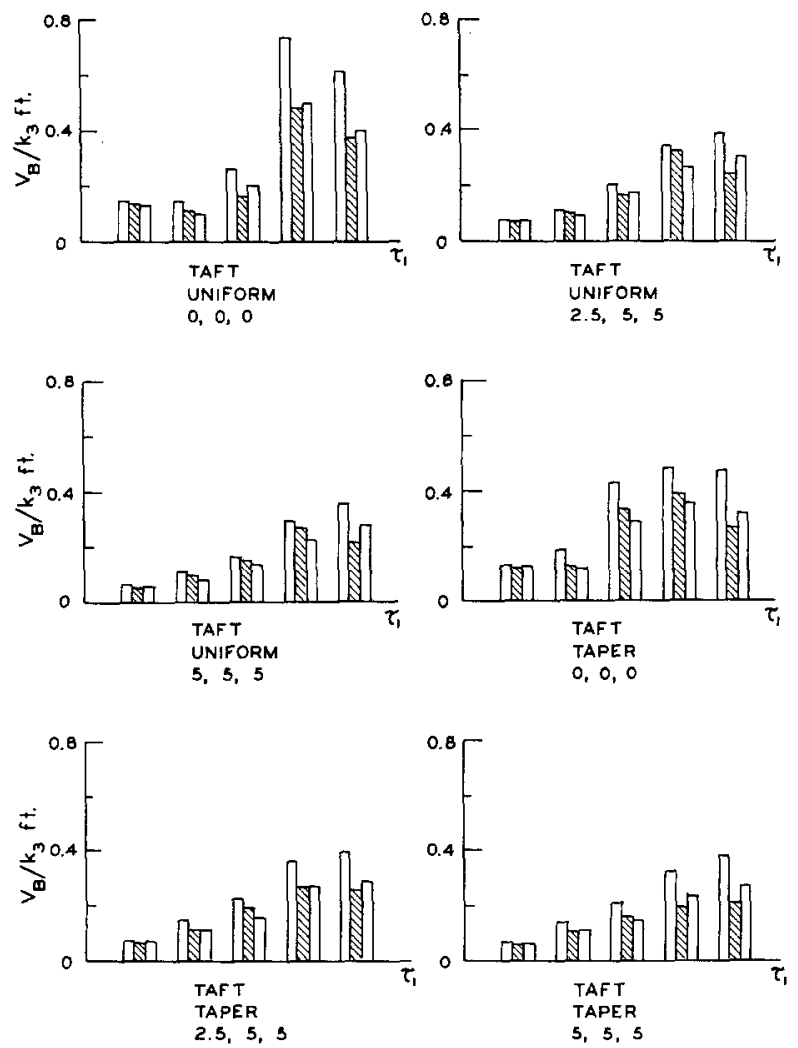

Fig. 6. Base shear comparisons (see fig. 4) Taft earthquake. 

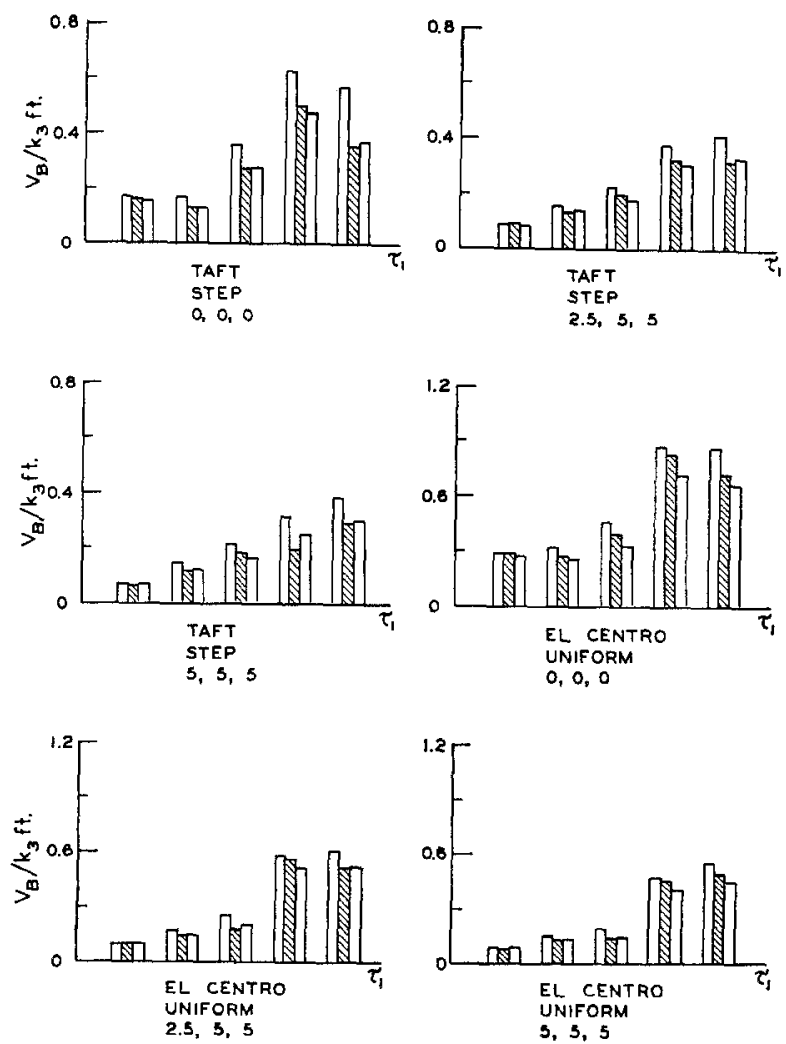

FIG. 7. Base shear comparisons (see fig. 4) Taft (cont.) and El Centro earthquake.
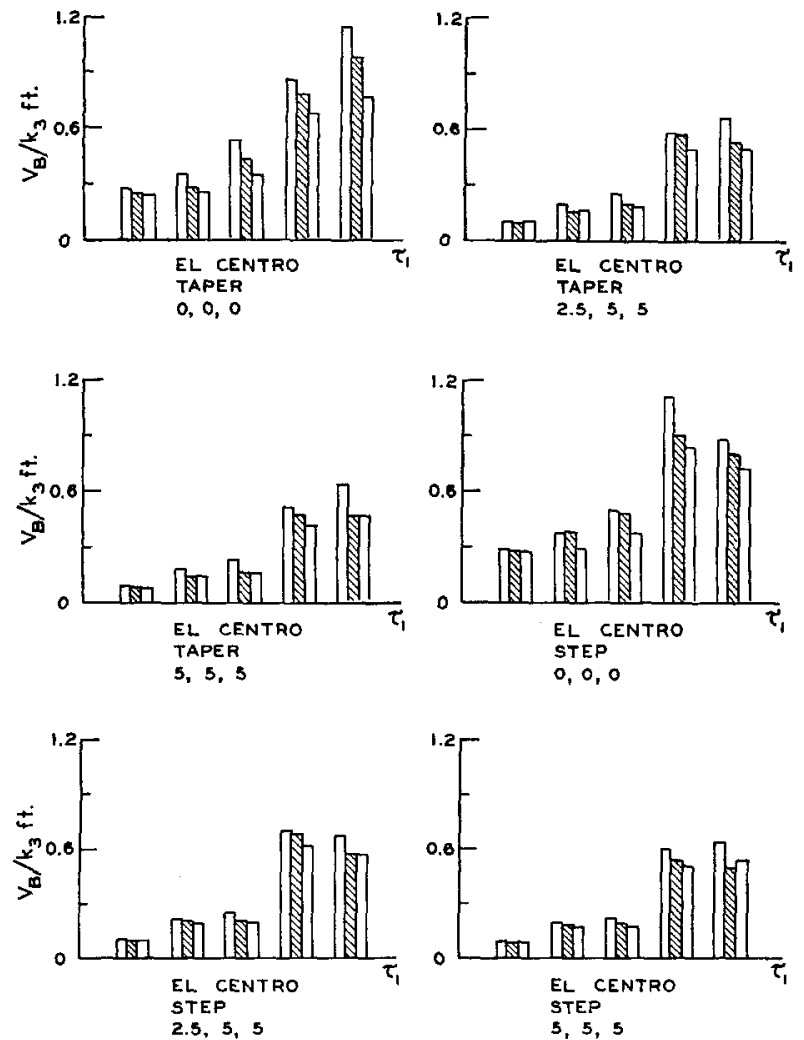

Fig. 8. Base shear comparisons (see fig. 4) El Centro earthquakes (cont.). 
strongly dependent upon the earthquake used, the type of structure, or the amount of damping. However, there does seem to be a tendency for the greatest deviations to occur at the longer periods of vibration.

The data from figs. 4-8 can be summarized in the form of the histograms of fig. 9 , which show the main conclusions of the investigation. These show the frequency of

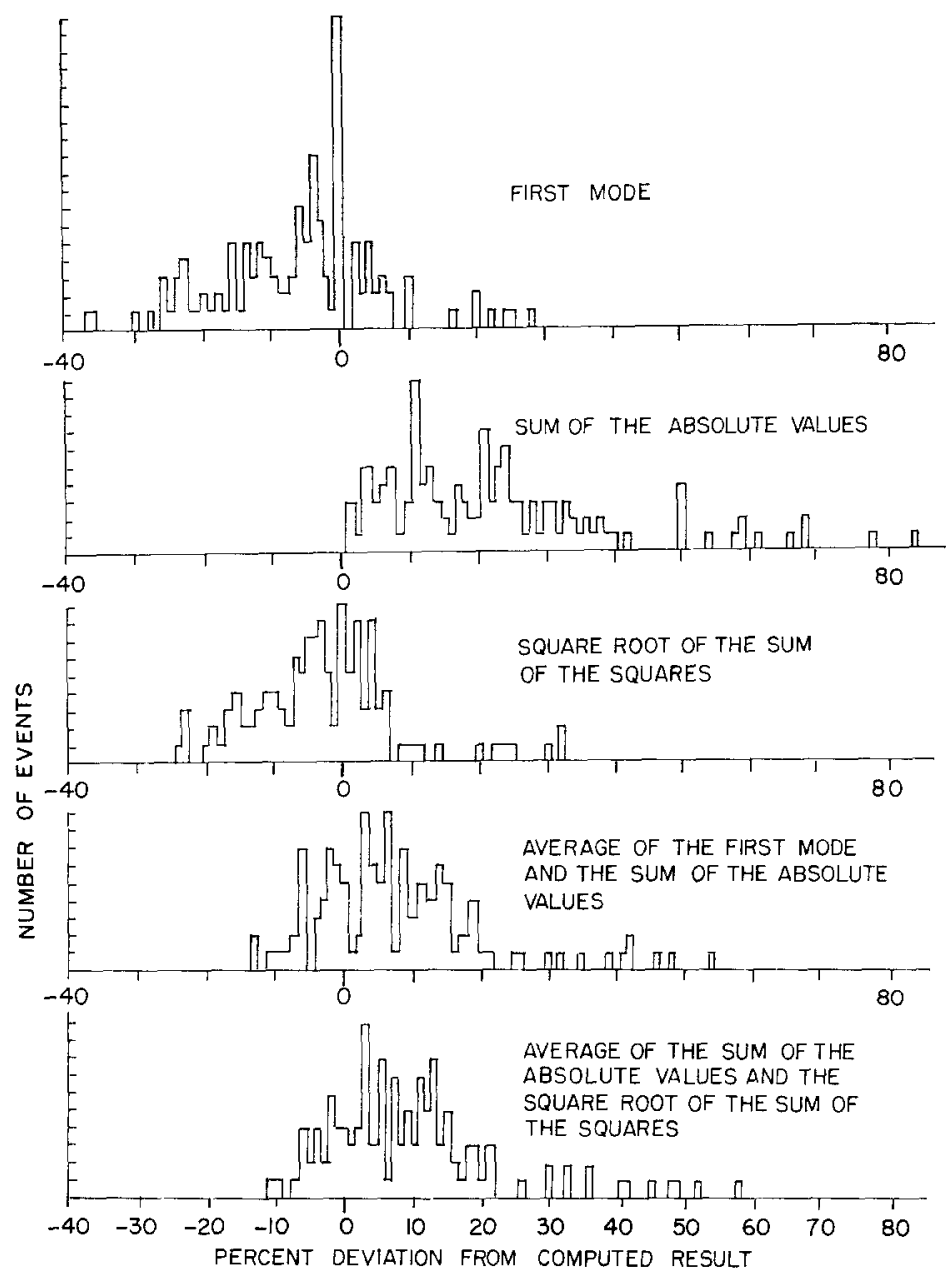

FIG. 9. Histograms of base shear deviations.

occurrence of the percent deviations from the "exact" computed results. The upper histogram exhibits the distribution of percent error if only the first mode shear is used. The second histogram shows the distribution of percent error when the sum of the absolute values is used. The third histogram is for the square root of the sum of the squares. The fourth histogram exhibits the results when the average of the first mode shear and the sum of the absolute values are compared with the "exact" value; and the fifth diagram is for the average of the sum of the absolute values and the square root of the sum of the squares. In interpreting fig. 9 reference should 
be made to figs. $4-8$, since the deviations do not necessarily follow the same patterns in all cases. For example, the largest deviations seem to occur at the longer periods, where the effects of the higher modes are expected to be relatively large.

\section{Results and Conclusions}

The significant properties of the histogram in fig. 9 are the location of the mean; the variance, or spread, of the results; and the range, or extreme values. It is interesting to note in fig. 9 that the response of the first mode only is close to the "exact" total response for a large number of cases. There are a significant number of combinations in which the first mode alone gives a larger result than the total response, because of a cancelling by the higher modes.

Referring to the histogram for the sum of the absolute values of the modes, two significant points are evident: (1) this is the only combination method that ensures that the response will not be underestimated; (2) although there are some cases which add up to sums much larger than the computed "exact" response, there are also a large number of combinations for which the absolute sum is within ten percent of the "exact" result. Since in a practical situation there is no way of knowing in advance where a particular structure-earthquake combination will fall on the histogram, this suggests that the absolute sum criterion may give closer results on the average than has sometimes been realized. For critical designs in which it might be essential to avoid the possibility of nonconservative deviations, the absolute sum criterion would seem to be most appropriate.

The histogram of the square root of the sum of the squares has a mean lying closest to zero and has the smallest range of values, however, this criterion will evidently lead to a large number of nonconservative deviations. It would seem that if the square root of the sum of the squares were to be used, suitable adjustments of seismic coefficient or of allowable stresses would be required to ensure adequate safety for all cases.

An additional method that might be used involves the averaging of the above criteria in such a way as to set prescribed limits to the permissible negative and positive deviations. This can be done by a system of weighted averages.

One useful weighted average can be defined in terms of the square root of the sum of the squares and the sum of the absolute values in the form:

$$
\text { Total Response }=\frac{\sqrt{a^{2}+b^{2}+c^{2}}+\cdots+m(a+b+c+\cdots)}{m+1}
$$

where $a, b, c, \cdots$ are the individual mode responses, and $m$ is the weighting factor. If it is supposed that the true response may be as high as $95 \%$ of the sum of the absolute values, and if it is assumed that three modes having contributions in the ratios $a: b: c=5: 3: 1$ are involved, which would be a realistic distribution for a typical shear type structure, it can be shown that the maximum value of the nonconservative (negative) deviation is given by (Merchant, 1961):

$$
\text { max. neg. deviation } \left.=\left[1-\frac{1}{0.95}\left(\frac{0.658}{m+1}+\frac{m}{m+1}\right)\right] 100 \text { (per cent }\right)
$$


If $m=0$, so that the square root of the sum of the squares criteria alone is used, it is found that the maximum nonconservative deviation is 30.7 percent. For a direct average, $m=1$, the maximum nonconservative deviation would be 12.8 percent. These values are seen to be consistent with the histograms of fig. 9 , which suggests that a sufficient number of cases have been included in the above investigations to yield realistic results.

By a suitable adjustment of the weighting factor $m$ the maximum nonconservative deviation could be limited to any desired level at the expense of an increased number of positive deviations.

The above conclusions must of course be limited to the types of structures and earthquake excitations investigated. If situations of a distinctly different type are encountered, additional studies will be required.

\section{ACKNOWLEDGMENTS}

Thanks are expressed to Professor G. W. Housner and T. K. Caughey of the Division of Engineering, California Institute of Technology, for their contributions to this investigation.

\section{REFERENCES}

Benioff, $\mathrm{H}$.

1934. "The Physical Evaluation of Seismic Destructiveness," Bull Seism. Soc. Amer.; $24: 398-403$

Biot, M. A.

1941. "A Mechanical Analyzer for the Prediction of Earthquake Stresses," Bull. Seism. Soc. Amer.; 31: 151-171.

1943. "Analytical and Experimental Methods in Engineering Seismology," Trans. A.S.C.E., Paper No. 2183, page 365, (Refer to discussion closure).

Caughey, T. K., D. E. Hudson, and R. V. Powell

1960. The C.I.T. Mark II Electric Analog Type Response Spectrum Analyzer for Earthquake Excitation Studies, Earthquake Engineering Research Laboratory, California Institute of Technology, Pasadena.

Clough, R. W.

1955. "On the Importance of Higher Modes of Vibration in the Earthquake Response of a Tall Building," Bull. Seism. Soc. Amer.; 45: 289-301.

Fung, Y. C., and M. V. Barton

1958. "Some Shock Spectra Characteristics and Uses," Jour. App. Mech.; 25: 365.

Housner, G. W.

1959. "Behavior of Structures During Earthquakes," Paper 2220, Engineering Mechanics Division, Proc. A.S.C.E.; 85: 107.

Housner, G. W., R. R. Martel, and J. L. Alford

1953. "Spectrum Analysis of Strong-Motion Earthquakes," Bull. Seism. Soc. Amer.; 43: $97-119$.

Hudson, D. E.

1960. "A Comparison of Theoretical and Experimental Determinations of Building Response to Earthquakes," Proc. Second World Conference on Earthquake Eng.; page 1105 .

1956. "Response Spectrum Techniques in Engineering Seismology," Proc. World Conference on Earthquake Eng., Earthquake Engineering Research Institute and the University of California, Berkeley, page 4-1.

Hudson, D. E., and G. W. Housner

1959. "Response Spectrum Analysis of the March 22, 1957 San Francisco Earthquakes," San Francisco Earthquakes of March 1957, Special Report 57, page 115, California Division of Mines, San Francisco. 
Jennings, R. L.

1958. "The Response of Multi-Storied Structures to Strong Ground Motion," MSc. Thesis, University of Illinois, Urbana.

Merehant, H. C.

1961. Mode Superposition Methods A pplied to Linear Mechanical Systems Under Earthquake Type Excitation, Earthquake Engineering Research Laboratory, California Institute of Technology, Pasadena.

Murphy, L. M., and W. K. Cloud

1954. United States Earthquakes-1952, Serial No. 773, U. S. Department of Commerce, Coast and Geodetic Survey, Washington, D. C.

Neumann, F.

1942. United States Earthquakes-1940, Serial No. 647, U. S. Department of Commerce, Coast and Geodetic Survey, Washington, D. C.

Rosenblueth, E.,

1956. "Some Applications of Probability Theory in Aseismic Design," Proc. World Conference on Earthquake Eng., page 8-1, Earthquake Engineering Research Institute and the University of California, Berkeley.

H. C. Merchant

Department of Mechanical Engineering

UNIVERSITY OF WASHINGTON

Seattle, Washington

D. E. Hudson

Division of Engineering

California Institute of Technologx

Pasadena, California

Manuseript received September 8. 1961. 